\title{
Production of multipartite entanglement for electron spins in quantum dots
}

\author{
F. Bodoky and M. Blaauboer \\ Kavli Institute of Nanoscience, Delft University of Technology, Lorentzweg 1, 2628 CJ Delft, The Netherlands
}

(Received 9 July 2007; published 13 November 2007)

\begin{abstract}
We propose how to generate genuine multipartite entanglement of electron spin qubits in a chain of quantum dots using the naturally available single-qubit rotations and two-qubit Heisenberg exchange interaction in the system. We show that the minimum number of required operations to generate entangled states of the GHZ, cluster, and W type scales linearly with the number of qubits and estimate the fidelities of the generated entangled cluster states. As the required single and two-qubit operations have recently been realized, our proposed scheme opens the way for experimental investigation of multipartite entanglement with electron spin qubits.
\end{abstract}

DOI: 10.1103/PhysRevA.76.052309 PACS number(s): 03.67.Mn, 03.67.Lx, 73.21.La, 03.65.Ud

\section{INTRODUCTION}

\section{A. Multipartite entanglement}

Bipartite entanglement refers to nonclassical correlations $[1,2]$ between two quantum particles, and multipartite entanglement to nonclassical correlations between three or more quantum particles. The characterization and quantification of the latter is far less understood than for bipartite entanglement [3]. In particular, in the case of multipartite entanglement it is no longer sufficient to ask if the qubits are entangled, but one needs to know how they are entangled as there are different ways-known as entanglement classes - in which three or more qubits can be entangled. For three qubits, there are two different equivalence classes of genuine tripartite entanglement [4], for four qubits already nine [5] or eight [6], and the number of classes is growing with the number of qubits. Two entangled states belong to the same equivalence class and are called locally equivalent if it is possible to transform between them using local operations and classical communication (LOCC) only, i.e., without interactions between two or more qubits. The two classes of entanglement for three qubits are the GHZ and the W class $[4,7]$, with representative members [8] $\left|\mathrm{GHZ}_{3}\right\rangle=\frac{1}{\sqrt{2}}(|000\rangle$ $+|111\rangle)$ and $\left|\mathrm{W}_{3}\right\rangle=\frac{1}{\sqrt{3}}(|001\rangle+|010\rangle+|100\rangle)$ (the subscript indicating the number of involved qubits), which are both going to be addressed in this paper. Both of these classes can be generalized to arbitrary numbers of qubits. Another interesting class of multipartite entanglement for four or more qubits is the cluster class [9], which forms the basis of proposals to implement a measurement-only quantum computer, the oneway quantum computing scheme [10]. These states maximize mutual bipartite entanglement and its four-partite representative is $\left|\phi_{4}\right\rangle=\frac{1}{2}(|0000\rangle+|0011\rangle+|1100\rangle-|1111\rangle)$.

Multiqubit entanglement is thus not a straightforward extension of bipartite entanglement and gives rise to new phenomena which can be exploited in quantum information and quantum computing processes. For example, there are quantum communication protocols that require multiparty entanglement such as universal error correction [11], quantum secret sharing [12], and telecloning [13]. Also, highly entangled multipartite states are needed for efficient quantum computing — all known quantum algorithms (such as Shor's factorization [14] and Grover's search [15] algorithm) work with multipartite entanglement — and GHZ states can be used to construct a universal quantum computer [16]. In addition, multiqubit entangled states provide a stronger test of local realism [17] which is based on individual (rather than statistical, as in the bipartite case) measurement results. As a general rule, one can say that the more particles are entangled, the more clearly nonclassical effects are exhibited and the more useful the states are for quantum applications.

So far, multipartite entanglement has been realized in a number of experiments, using liquid-state NMR [18], photons [19-22], cold atoms [23,24], and ions [25,26]. The latter two experiments for trapped ions have demonstrated the deterministic creation of a GHZ and a $W$ sate. Tripartite, and more generally multipartite, entanglement has not yet been realized for qubits in a solid-state environment. The latter type of qubit systems, consisting of, e.g., electrons confined in quantum dots [27] or superconducting Josephson junctions [28], are attractive since they are in principle scalable to an arbitrary number of qubits. A number of ideas have been suggested for the creation of tripartite entangled states, using exciton states in coupled quantum dots [29], electron-hole entanglement in the Fermi sea [30], and superconducting charge and flux qubits [31-33].

In this paper, we present schemes for deterministic creation of GHZ, $W$, and cluster states for electron spin qubits in quantum dots using the naturally available two-qubit (Heisenberg exchange) interaction and single-spin rotations. This choice of system is motivated by the fact that both single-qubit rotations and tunable two-qubit Heisenberg exchange interactions have already been demonstrated experimentally for these qubits $[34,35]$. However, our scheme can easily be used for other types of qubits as well, e.g., superconducting qubits for which tunable coupling has also very recently been realized [36]. We show that the required number of two-qubit interactions for the generation of $N$-partite entangled states and for the transformation from a disentangled to a maximally entangled basis scales linearly with $N$ for all types of entangled states considered here. We also present arguments that the total number of single- and twoqubit operations that our schemes predict is in fact the minimum number required to create these multipartite entangled states using single-qubit rotations and Heisenberg exchange interactions. 


\section{B. Electron spin qubits}

An electron spin qubit [37] consists of a single electron confined in a quantum dot (QD), an island in a semiconducting nanostructure [27]. The electron occupies discrete energy levels in the quantum dot which split into separate levels for spin up and spin down due to Zeeman splitting when the quantum dot is placed in an external magnetic field. The qubit is encoded in the spin degree of freedom, with the ground state spin up (denoted as $|\uparrow\rangle$ and defined along the direction of the magnetic field, which we assume to be the $z$ axis) corresponding to the logical bit $|0\rangle$ and spin down $(|\downarrow\rangle)$ corresponding to the logical bit $|1\rangle$. Electron spin qubits are attractive candidates for quantum computing since they are in principle scalable, relatively robust against decoherence (as compared to, e.g., charge qubits), and allow for a high level of control over individual qubits [38].

Two basic operations are available to manipulate the state of the qubit: First, coherent rotation of a spin around an axis in the $(x, y)$ plane using electron spin resonance (ESR), which consists of applying an oscillating time-dependent magnetic field $B(t)$ in this plane whose frequency is on resonance with the transition frequency between $|\uparrow\rangle$ and $|\downarrow\rangle$. A rotation around a certain angle is controlled by the time of application and the strength of the magnetic field, and is described by the evolution operator

$$
U_{R}(t)=\exp \left[(i \gamma / 2) \int_{0}^{t} B(\tau) \vec{k} \cdot \vec{\sigma} d \tau\right],
$$

which corresponds to the Hamiltonian $\mathcal{H}_{R}(t)$ $=-(1 / 2) \hbar \gamma B(t) \vec{k} \cdot \vec{\sigma}$, where $\gamma$ denotes the gyromagnetic ratio, $\vec{\sigma}=\left(\sigma_{x}, \sigma_{y}, \sigma_{z}\right)$, and $\vec{k} \equiv(\sin \theta \cos \phi, \sin \theta \sin \phi, \cos \theta)$ represents a unit vector on the Bloch sphere $[\theta \in[0, \pi), \phi \in[0,2 \pi)]$ in the direction of the magnetic field. The evolution operator (1) corresponds to a rotation $R_{k}^{(n)}(\beta)=\exp \left[-(1 / 2) i \beta \vec{k} \cdot \vec{\sigma}^{(n)}\right]$ of the $n$th qubit with angle $\beta$ $\equiv-\gamma \int_{0}^{t} B(\tau) d \tau$ around axis $\vec{k}$, where $\vec{\sigma}^{(n)} \equiv 1 \otimes \cdots \otimes \vec{\sigma} \otimes \cdots 1$. These ESR-induced rotations have recently been experimentally observed in quantum dots [34].

The second available operation is Heisenberg interaction between two spins described by the evolution operator $U_{E X}(t)=\exp (-i \hbar / 4) \int_{0}^{t} J(\tau) \vec{\sigma}^{(n)} \cdot \vec{\sigma}^{(n+1)} d \tau$. Here, $J(\tau)$ is the time-dependent exchange energy. By tuning the interaction time $t$ with a gate voltage, the (SWAP) ${ }^{\alpha}$ gate can be directly generated as $(\mathrm{SWAP})^{\alpha} \equiv U_{\mathrm{EX}}(t)$, and we will denote it from now on as

$$
\left(U_{\mathrm{SWAP}}\right)^{\alpha}=e^{-\alpha / 4 i \pi}\left[\begin{array}{cccc}
e^{\alpha / 2 i \pi} & 0 & 0 & 0 \\
0 & \cos \left(\frac{\alpha}{2} \pi\right) & i \sin \left(\frac{\alpha}{2} \pi\right) & 0 \\
0 & i \sin \left(\frac{\alpha}{2} \pi\right) & \cos \left(\frac{\alpha}{2} \pi\right) & 0 \\
0 & 0 & 0 & e^{\alpha / 2 i \pi}
\end{array}\right],
$$

where $\alpha(t) \equiv-\frac{\hbar}{\pi} \int_{0}^{t} J(\tau) d \tau$. For $\alpha=\frac{1}{2}$ the $\sqrt{U_{\text {SWAP }}}$ gate maximally entangles two spins of opposite directions. A $\sqrt{U_{\text {SWAP }}}$ operation has also recently been demonstrated for spin qubits [35]. Together, single-qubit rotations and the $\sqrt{U_{\mathrm{SWAP}^{-}} \text {gate }}$ form a universal set of quantum gates, into which any quantum operation can be decomposed [37].

\section{Outline}

This paper is organized as follows: in Sec. II we show how to generate $N$-partite entangled cluster states (Sec. II A), GHZ states (Sec. II B), and $W$ states (Sec. II C) using the smallest number of two-qubit $\left(U_{\text {SWAP }}\right)^{\alpha}$ operations and single-qubit rotations. In Sec. III we analyze the effects of errors in the timing of the $\left(U_{\text {SWAP }}\right)^{\alpha}$ - and single-qubit operations on the generation of $N$-partite cluster states, quantified by the fidelity. Finally, in Sec. IV, we discuss the feasibility of the multipartite entanglement generation scheme that we propose in the context of present-day experimental techniques, followed by conclusions.

\section{GENERATION OF MULTIPARTITE ENTANGLED STATES}

In this section we describe the generation of $N$-qubit entangled states in a chain of quantum dots where each dot is occupied by one electron, using single-qubit rotations and pairwise exchange interactions between nearest-neighbor spins. Starting from the ground state which consists of $N$ disentangled up-spins $|00 \cdots 0\rangle$ and using a recursive approach in $N$ we derive sequences of single-qubit rotations and two-qubit $\left(U_{\text {SWAP }}\right)^{\alpha}$ operations which, when applied to $|00 \cdots 0\rangle$, yield a $N$-partite cluster, GHZ, or $W$ state. We begin by briefly recounting the generation of entanglement and the implementation of a basis transformation for two qubits, and then present our main results in Secs. II A-II C below.

For two qubits, the shortest sequence required to transform the ground state $|00\rangle$ into a maximally entangled state is

$$
\sqrt{U_{\mathrm{SWAP}}^{(1,2)}} R_{\tilde{k}}^{(i)}(\pi)
$$

with $\tilde{k}$ an arbitrary axis in the $(x-y)$ plane and $i=1,2$.

It has also been shown that the shortest sequence required to implement the transformation from the standard (or computational) basis to a maximally entangled basis consisting of Bell states is given by [39]

$$
E_{\tilde{k}}^{(1,2, i)} \equiv \sqrt{U_{\mathrm{SWAP}}^{(1,2)}} R_{\tilde{k}}^{(i)}(\pi) \sqrt{U_{\mathrm{SWAP}}^{(1,2)}}
$$

with $i=1,2$. Two $\sqrt{U_{\text {SWAP }}}$ operations are needed in Eq. (4), since one $\sqrt{U_{\mathrm{SWAP}}}$-interaction only entangles two of the four standard basis states. Given a linear array of quantum dots in which each dot is occupied by a single spin qubit in the $|0\rangle$ or |1) state, entangled states of three or more qubits can be generated by pairwise application of the sequence (4), as we show in the next subsection. By applying local operations in between, one can control to which class the generated entangled states belong. This forms the basis of our calculations in the next two subsections. Without loss of generality, we choose the axis $\tilde{k}$ as $x$ and $i=1$ in Eq. (4), and omit the indices $\tilde{k}$ and $i$ in $E_{\tilde{k}}^{(1,2, i)}$ in the following.

\section{A. Cluster states}

It is straightforward to see that pairwise application of Eq. (4) to a chain of $N$ disentangled qubits, each of which are 
either in the $|0\rangle$ or $|1\rangle$ state, results in an $N$-partite entangled cluster state, as we prove in Proposition II.1 below. Specifically, we will prove that application of the sequence

$$
E_{C l}^{N} \equiv E^{(N-1, N)} \cdots E^{(1,2)}
$$

transforms an arbitrary disentangled state of the $N$-partite standard basis into a cluster state. The definition of linear cluster states for $N$ qubits is as follows: the cluster state is the state resulting when applying the Ising interaction $Z^{(n, n+1)}(\theta)=\exp (-i \theta / 4)\left(1-\sigma_{z}^{(n)}\right)\left(1-\sigma_{z}^{(n+1)}\right)$ with $\theta=\pi$ (the so-called $z$-phase gate) to each neighbor in a $N$-qubit chain prepared in the state $\otimes_{i=1}^{N} 1 / \sqrt{2}(|0\rangle+|1\rangle)$. The $z$-phase gate can be generated in quantum dots as $Z^{(n, n+1)}$ $=R_{z}^{(n)}(\pi / 2) R_{z}^{(n+1)}(-\pi / 2) \sqrt{U_{\text {SWAP }}^{(n, n+1)}} R_{z}^{(n)}(\pi) \sqrt{U_{\text {SWAP }}^{(n, n+1)}}$ (see [37]), where we omit the overall phase factor (as in the rest of this paper). In order to generate a cluster state, one thus has to apply this $z$-phase gate to each pair of qubits in the state $1 / \sqrt{2}(|0\rangle+|1\rangle)=R_{y}(\pi / 2)|0\rangle$. With these observations, we are now ready to prove the following.

Proposition II 1. The two sequences

$$
Z^{N} \equiv Z^{(N-1, N)} \cdots Z^{(1,2)} R_{y}^{(N)}\left(\frac{\pi}{2}\right) \cdots R_{y}^{(1)}\left(\frac{\pi}{2}\right)
$$

and

$$
E_{C l}^{N} \equiv E^{(N-1, N)} \cdots E^{(1,2)}
$$

are locally equivalent.

Proof. We start by rewriting the sequence $Z^{N}$ as

$Z^{N}=Z^{(N-1, N)} R_{y}^{(N)}\left(\frac{\pi}{2}\right) \cdots Z^{(2,3)} R_{y}^{(3)}\left(\frac{\pi}{2}\right) Z^{(1,2)} R_{y}^{(2)}\left(\frac{\pi}{2}\right) R_{y}^{(1)}\left(\frac{\pi}{2}\right)$

$$
=\widetilde{Z}^{(N-1, N)} \widetilde{Z}^{(N-2, N-1)} \cdots \widetilde{Z}^{(1,2)} R_{y}^{(1)}\left(\frac{\pi}{2}\right),
$$

which consists of $N-1$ applications of the operator $\widetilde{Z}^{(n, n+1)}$ $\equiv Z^{(n, n+1)} R_{y}^{(n+1)}(\pi / 2)$, plus an additional rotation $R_{y}^{(1)}(\pi / 2)$. We now write $\widetilde{Z}^{(n, n+1)}$ in terms of $E^{(n, n+1)}$ [Eq. (4)], with the goal to express $Z^{N}$ as $Z^{N} \equiv L E_{C l}^{N}$, where $L$ is a product of local operations. To this end, we use the identity

$$
\widetilde{Z}^{(1,2)} R_{y}^{(1)}\left(\frac{\pi}{2}\right)=R_{y}^{(1)}\left(\frac{\pi}{2}\right) R_{x}^{(1)}\left(\frac{\pi}{2}\right) R_{y}^{(2)}\left(\frac{\pi}{2}\right) R_{x}^{(2)}\left(-\frac{\pi}{2}\right) E^{(1,2),}
$$

and substitute Eq. (8) into Eq. (7b). The two rotations on qubit 1 commute with the sequence to the left of them, and can thus be absorbed into the local operation $L$, leaving $\widetilde{Z}^{(2,3)}$ acting on $R_{y}^{(2)}(\pi / 2) R_{x}^{(2)}(-\pi / 2)$. By rewriting

$$
\begin{aligned}
\tilde{Z}^{(n, n+1)} R_{y}^{(n)}\left(\frac{\pi}{2}\right) R_{x}^{(n)}\left(-\frac{\pi}{2}\right)= & R_{y}^{(n)}\left(\frac{\pi}{2}\right) R_{y}^{(n+1)}\left(\frac{\pi}{2}\right) \\
& \times R_{x}^{(n+1)}\left(-\frac{\pi}{2}\right) E^{(n, n+1)},
\end{aligned}
$$

repetitive substitution of Eq. (9) into Eq. (7b) for increasing $n$ and using commutation relations to reorder the resulting sequence such that rotations are shifted to the left of all $\sqrt{U_{\text {SWAP }}}$ operations, we find

$$
Z^{N}=L E^{(N-1, N)} \cdots E^{(1,2)}=L E_{C l}^{N},
$$

with

$$
\begin{aligned}
L= & R_{y}^{(N)}\left(\frac{\pi}{2}\right) R_{x}^{(N)}\left(-\frac{\pi}{2}\right) R_{y}^{(N-1)}\left(\frac{\pi}{2}\right) \cdots R_{y}^{(2)}\left(\frac{\pi}{2}\right) R_{y}^{(1)}\left(\frac{\pi}{2}\right) \\
& \times R_{x}^{(1)}\left(\frac{\pi}{2}\right) .
\end{aligned}
$$

Since $L$ does not change the entanglement class of the state that has been generated by $E_{C l}^{N}$, we have thus proven that application of the transformation $E^{N}$ to any state of the standard basis leads to a cluster state.

Inspecting the sequence $(6 \mathrm{~b})$, we see that $2(N-1) \sqrt{U_{\text {SWAP }}}$ operations and $(N-1)$ rotations are needed to generate a $N$-partite cluster state, whereas when using previously proposed implementations of the $z$-phase gate $Z^{(n, n+1)}$ [40] a total of $(4 N-3)$ rotations are required. The sequences given by Eqs. (6a) and (6b) transform the standard basis into a basis of cluster states. Note that in order to transform the ground state $|0 \cdots 0\rangle$ into a cluster state, the sequence $E^{(N-1, N)} \cdots E^{(2,3)} \sqrt{U_{\mathrm{SWAP}}^{(1,2)}} R_{x}^{(1)}(\pi)$, which contains one $\sqrt{U_{\text {SWAP }}}$ operation less than Eq. (6b), is sufficient, since $\sqrt{U_{\mathrm{SWAP}}^{(1,2)}} R_{x}^{(1)}(\pi)$ [see Eq. (3)] already maximally entangles the first two qubits.

A special property of the $z$-phase gate, being a diagonal matrix, is that the $Z^{(n, n+1)}$ matrices commute for different $n$. As a result, the transformation (6a) and (6b) from the standard basis to the cluster basis can be done in two steps: first, all the even-numbered qubits are simultaneously entangled to their (odd) neighbor to the right, and then the same is done for the odd-numbered qubits. In the next proposition, we show that the same commutation relation applies for the (nondiagonal) $E^{(n, n+1)}$ matrices in Eq. (5).

Proposition II 2. The two sequences

$$
\begin{aligned}
& Z^{(N-1, N)} Z^{(N-3, N-2)} \cdots Z^{(3,4)} Z^{(1,2)} Z^{(N-2, N-1)} \cdots Z^{(2,3)} \\
& \quad \times R_{y}^{(N)}\left(\frac{\pi}{2}\right) \cdots R_{y}^{(1)}\left(\frac{\pi}{2}\right)
\end{aligned}
$$

and

$$
E^{(N-1, N)} E^{(N-3, N-2)} \cdots E^{(1,2)} E^{(N-2, N-1)} \cdots E^{(2,3)}
$$

are locally equivalent.

Proof. Since the $Z^{(n, n+1)}$ matrices commute for different $n$, the sequence (12a) is equivalent to the right-hand side of Eq. (6a) and hence by Proposition II.1 to the sequence (6b). It now remains to be shown that (6b) is equivalent to $(12 b)$. This directly follows from the fact that the matrices $E^{(n, n+1)}$ and $E^{(n+1, n+2)}$ [Eq. (4)] commute

$$
\left[E^{(n, n+1)}, E^{(n+1, n+2)}\right]=0 .
$$

The two sequences (12a) and (12b) differ by the same overall local operation $L$ [Eq. (11)] as in Proposition II 1. 
Propositions II 1 and II 2 imply that the sequences (6a) and (12a), which consist only of the entangling operations $E^{(n, n+1)}$, create a state of the cluster class. Can this be done with fewer operations? To answer this question, consider first three qubits: we have already seen that the first $\sqrt{U_{\text {SWAP }}}$ operation in the sequence (6b) can be omitted when entangling the ground state, and if we start in an appropriate excited state also the first rotation is not needed. That leaves the sequence $\sqrt{U_{\mathrm{SWAP}}^{(1,2)}} R_{x}^{(1)} \sqrt{U_{\mathrm{SWAP}}^{(1,2)}} \sqrt{U_{\mathrm{SWAP}}^{(2,3)}}$. It is straightforward to check, e.g., by calculating the tangle $\tau$ [42] of the resulting entangled state, that this is the shortest sequence of $\sqrt{U_{\text {SWAP }}}$ operations and single qubit rotations that creates a tripartite cluster state: if any of the four operations is omitted, $\tau=0$ and the resulting state is no longer a cluster state. Generalizing to an arbitrary number of qubits, we note that omitting any operation in the sequence (6b) or (12b) leads to a state which is not maximally connected in the sense of Ref. [9], and therefore cannot be a cluster state.

\section{B. GHZ states}

In this section we show how the disentangled $N$-qubit state $|00 \cdots\rangle$ can be transformed into a $N$-qubit GHZ state using single-qubit rotations and $\sqrt{U_{\mathrm{SWAP}^{-}}}$-operations. We start with the observation that GHZ states are generated by successive application of the controlled-NOT (CNOT) gate $U_{\mathrm{CNOT}}$ :

Observation 1. Starting from the disentangled $N$-qubit state $|00 \cdots 0\rangle$, the $N$-partite $\mathrm{GHZ}$ state $\left|\mathrm{GHZ}_{N}\right\rangle=|00 \cdots 0\rangle$ $+|11 \cdots 1\rangle$ (disregarding normalization) is generated by $N$ -2 applications of the $U_{\mathrm{CNOT}}$,

$$
\begin{aligned}
\left|\mathrm{GHZ}_{N}\right\rangle= & \left(\prod_{n=N-1}^{2} U_{\mathrm{CNOT}}^{(n, n+1)}\right) R_{y}^{(1)}\left(-\frac{\pi}{2}\right) R_{x}^{(1)}\left(\frac{\pi}{2}\right) \\
& \times R_{x}^{(2)}\left(\frac{\pi}{2}\right) \sqrt{U_{\mathrm{SWAP}}^{(1,2)}} R_{y}^{(1)}(\pi)|00 \cdots 0\rangle,
\end{aligned}
$$

where $U_{\mathrm{CNOT}}^{(n, n+1)}$ denotes a $U_{\mathrm{CNOT}}$ operation with the $n$th qubit as control bit, and the $(n+1)$ th qubit as target bit.

Note that the order in the product in Eq. (14), starting with the highest $n=N-1$, is essential. In Eq. (14), the operation $R_{y}^{(1)}\left(-\frac{\pi}{2}\right) R_{x}^{(1)}\left(\frac{\pi}{2}\right) R_{x}^{(2)}\left(\frac{\pi}{2}\right) \sqrt{U_{\text {SWAP }}^{(1,2)}} R_{y}^{(1)}(\pi)$ on the first two qubits yields the Bell state $(1 / \sqrt{2})(|00\rangle+|11\rangle)$ and each successive $U_{\mathrm{CNOT}}$ gate entangles one more qubit to this superposition, resulting in the $N$ partite GHZ state $\left|\mathrm{GHZ}_{N}\right\rangle$. Using single-spin rotations and $\sqrt{U_{\text {SWAP }}}$ operations only, the shortest sequence of operations required to implement the $U_{\mathrm{CNOT}}$ gate is given by [41]

$$
\begin{aligned}
U_{\mathrm{CNOT}}^{(n, n+1)} \equiv & R_{y}^{(n)}\left(-\frac{\pi}{2}\right) R_{x}^{(n)}\left(-\frac{\pi}{2}\right) R_{x}^{(n+1)}\left(\frac{\pi}{2}\right) \sqrt{U_{\mathrm{SWAP}}^{(n, n+1)}} \\
& \times R_{x}^{(n)}(\pi) \sqrt{U_{\mathrm{SWAP}}^{(n, n+1)}} R_{y}^{(n)}\left(\frac{\pi}{2}\right) .
\end{aligned}
$$

We now substitute Eq. (15) into Eq. (14). By moving all single-qubit rotations that commute with the sequence of operations to the left of them in front of all $\sqrt{\mathrm{SWAP}}$ operations and defining

$$
\widetilde{U_{\mathrm{CNOT}}^{(n, n+1)}} \equiv \sqrt{U_{\mathrm{SWAP}}^{(n, n+1)}} R_{x}^{(n)}(\pi) \sqrt{U_{\mathrm{SWAP}}^{(n, n+1)}} R_{y}^{(n)}\left(\frac{\pi}{2}\right) R_{x}^{(n)}\left(\frac{\pi}{2}\right),
$$

Eq. (14) becomes

$$
\begin{gathered}
\left|\mathrm{GHZ}_{N}\right\rangle=\widetilde{L}\left(\prod_{n=N-1}^{2} \widetilde{U_{\mathrm{CNOT}}^{(n, n+1)}}\right) \sqrt{U_{\mathrm{SWAP}}^{(1,2)}} R_{y}^{(1)}(\pi)|00 \cdots 0\rangle, \\
N \geq 3,
\end{gathered}
$$

where $\tilde{L}$ consists of single-qubit rotations. We see from Eq. (17) that a total of $(2 N-3) \sqrt{U_{\text {SWAP }}}$ operations and a minimum of $(3 N-5)$ single-qubit rotations are needed to transform the separable state $|00 \cdots\rangle$ into an $N$-partite entangled state in the GHZ class. Compared to cluster states (see the previous section), the generation of a GHZ state thus requires $(2 N-4)$ more single-qubit rotations. In practice, implementation of the sequence (17) can be done in the most efficient way by applying two $\sqrt{U_{\text {SWAP }}}$ operations simultaneously in each step. This can be achieved by starting with qubit number $m \equiv N / 2$ (for $N$ even, or $m \equiv(N+1) / 2$ for $N$ odd) in the middle of the chain and reordering the sequence (17) as (for $N$ even):

$$
\prod_{j=N-1}^{m+1}\left(\widetilde{U_{\mathrm{CNOT}}^{(N-j+1, N-j)}} \widetilde{U_{\mathrm{CNOT}}^{(j, j+1)}}\right) \sqrt{U_{\mathrm{SWAP}}^{(m, m+1)}} R_{y}^{(m)}(\pi)|00 \cdots 0\rangle .
$$

The two $\overparen{U_{\mathrm{CNOT}}}$ operations in between the brackets in Eq. (18) can be performed simultaneously. An analogous expression as Eq. (18) applies if $N$ is odd.

Using the same line of reasoning as in the previous subsection and the fact that GHZ states are also maximally connected [9], one can directly show that the number of $\sqrt{U_{\text {SWAP }}}$ operations and the number of rotations over $\pi$ in Eq. (17) is minimal. Although we have no formal proof for this, we suspect that the total number of rotations in Eq. (17) is minimal.

\section{C. $W$ states}

A $W$ state for $N$ qubits is a multipartite entangled state that can be written in the form

$$
\left|W_{N}\right\rangle=\frac{1}{\sqrt{N}}(\underbrace{(|10 \cdots 0\rangle+|01 \cdots 0\rangle+\cdots+|00 \cdots 1\rangle}_{N \text { terms }}) .
$$

In this section we show how $\left|W_{N}\right\rangle$ can be generated using the least possible number of single-qubit rotations and $\sqrt{U_{\text {SWAP }}}$ operations when starting from $N$ disentangled qubits in the state $|00 \cdots 0\rangle$. Since the $W$ state (19) consists of a superposition of $N$-qubit states in which one bit value differs from all the others (representing, for example, a collective spin state of $N$ spins with one distributed excitation), a straightforward "recipe" to generate a $N$-partite $W$ state starting from the ground state $|00 \cdots 0\rangle$ is to flip one qubit to the value " 1 " and then distribute this bit value equally over all 
qubits such that each qubit is excited with a fraction $1 / N$. Rotating qubit 1 and then applying a $\left(U_{\mathrm{SWAP}}\right)^{\alpha}$ interaction to qubits 1 and 2 results in the fraction of the first excitation being $\cos (\alpha / 2)$; so in order to have the first qubit excited with a fraction $1 / N, \alpha$ has to be chosen as $\alpha$ $=2 \arccos (\sqrt{1 / N})$. By applying the same reasoning to all the following qubits, we find

$$
\begin{gathered}
\left|W_{N}\right\rangle=J^{(N-1, N)}\left(\mu_{N-1}\right) \cdots J^{(1,2)}\left(\mu_{1}\right) R_{y}^{(1)}(\pi)|00 \cdots 0\rangle, \\
\text { with } \mu_{n}=\frac{2}{\pi} \arccos \left(\sqrt{\frac{1}{N-n+1}}\right),
\end{gathered}
$$

where $J^{(n, n+1)}(\mu)$ denotes a $\left(U_{\text {SWAP }}\right)^{\mu}$ gate on the qubits $n$ and $n+1$.

For implementation of the sequence (20) we can apply the same trick as for the GHZ states by starting from the middle qubit in the two opposite directions along the chain and performing two $\sqrt{U_{\text {SWAP }}}$ operations simultaneously. To find the correct interaction angles we then need to distinguish between even and odd number of qubits $N$. For the even case, we rotate the qubit $m=N / 2$, apply an interaction $J^{(m, m+1)}(1 / 2)$, and then proceed as with two independent strings of length $m$. For the odd case, we rotate the qubit $m=(N+1) / 2$, apply an interaction $J^{(m, m+1)}(\mu)$ with $\mu$ $=2 \arccos (\sqrt{m / N})$, and then proceed as with two independent strings of length $m$ (to the left) and $m-1$ (to the right). Thus the required number of operations to transform the state $|00 \cdots 0\rangle$ into a $N$-partite $W$ state consists of $(N-1)$ $\left(U_{\text {SWAP }}\right)^{\mu}$-operations and one rotation.

\section{FIDELITY}

In the previous sections we have assumed perfect control of the single- and two-qubit operations, i.e., we assumed that all the pulses were perfectly timed. However, for a physical implementation it is important to estimate the effect of imperfections in the $\left(U_{\mathrm{SWAP}}\right)^{\alpha}$ gate operations and in singlequbit rotations on the intended final entangled states. In this section, we provide such an estimate for the generation of cluster states [Eq. (5)], assuming that the control of each

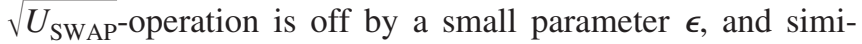
larly for each rotation by a small parameter $\delta$, i.e., we replace $\sqrt{U_{\mathrm{SWAP}}} \rightarrow\left(U_{\mathrm{SWAP}}\right)^{(1 / 2+\epsilon)} \quad$ and $\quad R(\pi) \rightarrow R(\pi+\delta)$, where $|\epsilon| \ll 1 / 2$ and $|\delta| \ll \pi$. As measure for the effect of the inaccuracies $\epsilon$ and $\delta$ we use the fidelity $F$ [43], which describes the overlap between the intended ("perfect") state $\left|\phi_{N}\right\rangle$ and the generated (in the presence of the inaccuracies) state $\left|\widetilde{\phi}_{N}\right\rangle$. For pure states, $F$ is defined as:

$$
F \equiv \sqrt{\left\langle\phi_{N} \mid \widetilde{\phi}_{N}\right\rangle\left\langle\phi_{N} \mid \phi_{N}\right\rangle}
$$

where $\left|\widetilde{\phi}_{N}\right\rangle$ and $\left|\phi_{N}\right\rangle$ are both normalized.

In the following, we calculate $F$ as a function of $N$ for the cluster state

$$
\left|\phi_{N}\right\rangle=E^{(N-1, N)} \cdots E^{(1,2)}|00 \cdots\rangle,
$$

up to second order in $\epsilon$ and $\delta$. We start by expanding $\left(U_{\mathrm{SWAP}}\right)^{1 / 2+\epsilon}$ [Eq. (2)] and $R_{x}(\pi+\delta)$ [Eq. (1)] up to second order in $\delta$ and $\epsilon$ :

$$
\begin{gathered}
\left(U_{\mathrm{SWAP}}\right)^{1 / 2+\epsilon}=e^{-i \pi / 4(1 / 2+\epsilon)}\left[\begin{array}{cccc}
e^{i \pi / 2(1 / 2+\epsilon)} & 0 & 0 & 0 \\
0 & \cos \left[\frac{\pi}{2}\left(\frac{1}{2}+\epsilon\right)\right] & i \sin \left[\frac{\pi}{2}\left(\frac{1}{2}+\epsilon\right)\right] & 0 \\
0 & i \sin \left[\frac{\pi}{2}\left(\frac{1}{2}+\epsilon\right)\right] & \cos \left[\frac{\pi}{2}\left(\frac{1}{2}+\epsilon\right)\right] & 0 \\
0 & 0 & 0 & e^{i \pi / 2(1 / 2+\epsilon)}
\end{array}\right], \\
R_{x}[\pi+\delta]=\left[\begin{array}{cccc}
\cos \left(\frac{\pi+\epsilon}{2}\right) & 0 & -i \sin \left(\frac{\pi+\epsilon}{2}\right) & 0 \\
0 & \cos \left(\frac{\pi+\epsilon}{2}\right) & 0 & -i \sin \left(\frac{\pi+\epsilon}{2}\right) \\
-i \sin \left(\frac{\pi+\epsilon}{2}\right) & 0 & \cos \left(\frac{\pi+\epsilon}{2}\right) & 0 \\
0 & -i \sin \left(\frac{\pi+\epsilon}{2}\right) & 0 & \cos \left(\frac{\pi+\epsilon}{2}\right)
\end{array}\right]
\end{gathered}
$$

Since we assume $|\epsilon|$ and $|\delta|$ to be small compared to $1 / 2$ and $\pi$, respectively, we can expand Eqs. (23) and (24) to second order in $\epsilon$ and $\delta$ :

$$
\begin{gathered}
\exp \left(\frac{i \pi}{4}+\frac{i \pi}{2} \epsilon\right)=\exp \left(\frac{i \pi}{4}\right) \exp \left(\frac{i \pi}{2} \epsilon\right)=c\left(1+i A-\frac{A^{2}}{2}+O\left(A^{3}\right)\right), \\
\sin \left(\frac{\pi}{4}+\frac{\pi}{2} \epsilon\right)=\sin \left(\frac{\pi}{4}\right) \cos \left(\frac{\pi}{2} \epsilon\right)+\cos \left(\frac{\pi}{4}\right) \sin \left(\frac{\pi}{2} \epsilon\right)=\frac{1}{\sqrt{2}}\left(1+A-\frac{A^{2}}{2}+O\left(A^{3}\right)\right), \\
\cos \left(\frac{\pi}{4}+\frac{\pi}{2} \epsilon\right)=\cos \left(\frac{\pi}{4}\right) \cos \left(\frac{\pi}{2} \epsilon\right)-\sin \left(\frac{\pi}{4}\right) \sin \left(\frac{\pi}{2} \epsilon\right)=\frac{1}{\sqrt{2}}\left(1-A-\frac{A^{2}}{2}+O\left(A^{3}\right)\right),
\end{gathered}
$$




$$
\begin{gathered}
\sin \left(\frac{\pi}{2}+\frac{\delta}{2}\right)=\sin \left(\frac{\pi}{2}\right) \cos \left(\frac{\delta}{2}\right)+\cos \left(\frac{\pi}{2}\right) \sin \left(\frac{\delta}{2}\right)=1-\frac{B^{2}}{2}+O\left(B^{3}\right), \\
\cos \left(\frac{\pi}{2}+\frac{\delta}{2}\right)=\cos \left(\frac{\pi}{2}\right) \cos \left(\frac{\delta}{2}\right)-\sin \left(\frac{\pi}{2}\right) \sin \left(\frac{\delta}{2}\right)=-B+O\left(B^{3}\right) .
\end{gathered}
$$

Here $c \equiv \exp (i \pi / 4)$ is a constant, $A \equiv \pi \epsilon / 2$, and $B \equiv \delta / 2$. Using Eqs. (23), (24), and (25a)-(25e), we construct the entangling operation $\widetilde{E} \equiv\left(U_{\mathrm{SWAP}}\right)^{1 / 2+\epsilon} R_{x}^{(1)}(\pi+\delta)\left(U_{\mathrm{SWAP}}\right)^{1 / 2+\epsilon}$ up to second order in the parameters $\epsilon$ and $\delta$ :

$$
\tilde{E}=\left[\begin{array}{ccccc}
-c(1+2 i A) B & \frac{1}{\sqrt{2}}\left[1+(1+i) A-(1-i) A^{2}-\frac{B^{2}}{2}\right] & \ldots \ldots & \frac{-i}{\sqrt{2}}\left[1-(1-i) A-(1+i) A^{2}-\frac{B^{2}}{2}\right] & 0 \\
\frac{1}{\sqrt{2}}\left[1+(1+i) A-(1-i) A^{2}-\frac{B^{2}}{2}\right] & -2 c^{3} A B & \ldots \ldots & -c B & \frac{-i}{\sqrt{2}}\left[1-(1-i) A-(1+i) A^{2}-\frac{B^{2}}{2}\right] \\
\frac{-i}{\sqrt{2}}\left[1-(1-i) A-(1+i) A^{2}-\frac{B^{2}}{2}\right] & -c B & \ldots \ldots & -2 c^{3} A B & \frac{1}{\sqrt{2}}\left[1+(1+i) A-(1-i) A^{2}-\frac{B^{2}}{2}\right] \\
0 & \frac{-i}{\sqrt{2}}\left[1-(1-i) A-(1+i) A^{2}-\frac{B^{2}}{2}\right] & \ldots & \frac{1}{\sqrt{2}}\left[1+(1+i) A-(1-i) A^{2}-\frac{B^{2}}{2}\right] & -c(1+2 i A) B
\end{array}\right] .
$$

The cluster state $\left|\widetilde{\phi}_{N}\right\rangle$ is defined as

$$
\left|\widetilde{\phi}_{N}\right\rangle=\widetilde{E}^{(N-1, N)} \cdots \widetilde{E}^{(1,2)}|0 \cdots 0\rangle \text {. }
$$

The order of the entangling operations $\widetilde{E}^{(n, n+1)}$ in Eq. (27) has to be the same as for the intended state $\left|\phi_{N}\right\rangle$ in Eq. (22), since different ordering generates different states (although in the same entanglement class). We now calculate $\left|\phi_{N}\right\rangle$ and $\left|\widetilde{\phi}_{N}\right\rangle$ and from these the fidelity $F[\mathrm{Eq} .(21)]$ for an increasing number of qubits. We then find [44]

$$
F=\sqrt{1-(N-1) A^{2}-\frac{5 N-9}{2} B^{2}}, \quad N \geq 3,
$$

for $(N-1) A^{2}-(5 N-9) B^{2} / 2 \leq 1$. We have verified that Eq. (28) is valid up to $N=10$, and suspect that it is true for all values of $N$. We remark that the fidelity $F$ increases as the square root of $N$, the number of qubits. Figure 1(a) shows the fidelity as a function of the inaccuracies $\epsilon$ and $\delta$ for the case of three qubits. Numerical evaluation shows remarkably high fidelities even for systems with many qubits, e.g., $F(N=10, \epsilon=0.05, \delta=0.1)=0.95$. This suggests that the proposed sequences for the cluster states enable generation of many-qubit entangled states with high fidelity. Although the fidelity of generating, e.g., $N$-partite GHZ states (not shown) are lower because there are more single-qubit rotations required to transform the $N$-qubit ground state into a GHZ state, they are also within reach of experimental implementation, as we discuss in the next section.

\section{DISCUSSION}

In this section we briefly discuss the experimental feasibility of generating multipartite entangled states of electron spins in quantum dots. As demonstrated experimentally, the duration of a $\sqrt{U_{\text {SWAP }}}$ operation of two electron spins is $\sim 180$ ps [35], and a spin rotation over $\pi / 2$ requires $\sim 27 \mathrm{~ns}$ [34]. As a rough estimate, we then find that the sequence Eq. (12b) to implement a $N$-partite cluster state using simultaneous application of $\sqrt{U_{\text {SWAP }}}$ operations to all pairs of qubits requires $\sim 2 \times 50=100 \mathrm{~ns}$ for any $N$ (since the linear array of qubits can be entangled in just two steps: first each of the even-numbered qubits to their right neighbor, and then the same for the odd-numbered qubits). The time required to implement the $N$-partite GHZ state (17) depends on the num-
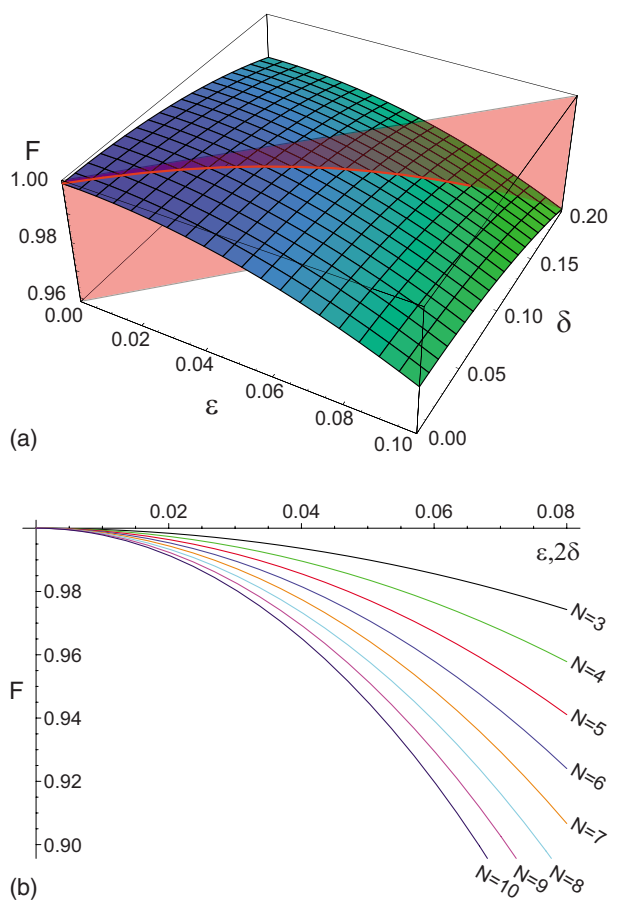

FIG. 1. (Color online) (a) Three-dimensional (3D) plot of the fidelity $F$, Eq. (28), for $N=3$ as a function of the two parameters $\epsilon$ and $\delta$. The red line and the transparent red plane show the cut along which the 2D plots in part (b) are taken. (b) The decrease of the fidelity $F$ when the number of qubits $N$ is increased. We chose here $\delta=\epsilon / 2$. 
ber of qubits and amounts to $\sim \frac{(N-1)}{2} \times 100 \mathrm{~ns}$ [using the ordering given in Eq. (18)]. The limiting time for the implementation of these sequences of operations is the decoherence time $T_{2}$, which has not yet been measured for a single spin. Rabi oscillations of a single electron spin [34] have been seen for more than $1 \mu$ s, indicating a decoherence time $T_{2} \gtrsim 1 \mu \mathrm{s}$. Based on this estimate for $T_{2}$, the generation of a $N$-partite cluster state and a $N$-partite $W$ state thus seems feasible for any $N$ in a time shorter than $T_{2}$, whereas the generation of GHZ states should be possible for up to $\sim 10$ qubits [45].

To conclude, we have calculated general sequences to generate genuine $N$-partite entangled states in various entanglement classes starting from a separable $N$-qubit state in the computational basis and using the least possible number of single-qubit rotations and two-qubit exchange interactions. For all entangled states that we considered (cluster states, GHZ states and $W$ states) we find that the total number of operations required to generate these states scales linearly with the number of qubits $N$. The generation of $N$-partite $W$ states requires the least amount of operations, namely $(N-1) \sqrt{U_{\text {SWAP }}}$ operations and one rotation. They are followed by the $N$-partite cluster states that require a mini- mum of $(2 N-3)$ exchange interactions and $(N-1)$ rotations and the GHZ states that also require a minimum of $(2 N-3)$ exchange interactions and $(3 N-5)$ single-qubit rotations. We also calculated the fidelity $F$ for the generation of $N$-partite cluster states in the presence of imperfect single-qubit rotations and $\sqrt{U_{\text {SWAP }}}$ operations, and find that $F$ decreases as $F \sim \sqrt{1-\mu N+\nu}$ as the number of qubits grows, with $\mu, \nu$ $>0$, and $\mu N-\nu \leq 1$.

Our results can be implemented for electron spins in quantum dots [46], for which Heisenberg exchange is the naturally available two-qubit interaction in the system. We estimate that our proposed scheme for the generation of multipartite entangled states is feasible for at least ten qubits within current experimental accuracy. Finally, we emphasize that the approach used in this paper can be used for any kind of two-qubit entangling interaction and provides an analytical scheme to calculate the implementation of multipartite entangled states for any type of qubit.

\section{ACKNOWLEDGMENTS}

This research was supported by the Netherlands Organisation for Scientific Research (NWO).
[1] A. Einstein, B. Podolski, N. Rosen, Phys. Rev. 47, 777 (1935).

[2] J. S. Bell, Physics (Amsterdam) 1, 195 (1964).

[3] R. Horodecki, P. Horodecki, M. Horodecki, K. Horodecki, e-print arXiv:quant-ph/0702225.

[4] W. Dür, G. Vidal, and J. I. Cirac, Phys. Rev. A 62, 062314 (2000)

[5] F. Verstraete, J. Dehaene, B. De Moor, and H. Verschelde, Phys. Rev. A 65, 052112 (2002).

[6] L. Lamata, J. Leon, D. Salgado, and E. Solano, Phys. Rev. A 75, 022318 (2007).

[7] A. Acín, D. Bruß, M. Lewenstein, and A. Sanpera, Phys. Rev. Lett. 87, 040401 (2001).

[8] D. Greenberger, M. A. Horne, and A. Zeilinger, in Bell's Theorem, Quantum Theory, and Conceptions of the Universe, edited by M. Kafatos (Kluwer, Dordrecht, 1989), pp. 69-72; D. M. Greenberger, M. A. Horne, A. Shimony, and A. Zeilinger, Am. J. Phys. 58, 1131 (1990).

[9] H. J. Briegel and R. Raussendorf, Phys. Rev. Lett. 86, 910 (2001).

[10] R. Raussendorf and H. J. Briegel, Phys. Rev. Lett. 86, 5188 (2001).

[11] P. W. Shor, Phys. Rev. A 52, R2493 (1995); R. Laflamme, C. Miquel, J. P. Paz, and W. H. Zurek, Phys. Rev. Lett. 77, 198 (1996); C. H. Bennett, D. P. DiVincenzo, J. A. Smolin, and W. K. Wootters, Phys. Rev. A 54, 3824 (1996).

[12] M. Hillery, V. Bužek, and A. Berthiaume, Phys. Rev. A 59, 1829 (1999).

[13] M. Murao, D. Jonathan, M. B. Plenio, and V. Vedral, Phys. Rev. A 59, 156 (1999); S. Koike, H. Takahashi, H. Yonezawa, N. Takei, S. L. Braunstein, T. Aoki, and A. Furusawa, Phys. Rev. Lett. 96, 060504 (2006).

[14] P. W. Shor, SIAM J. Comput. 26, 1484 (1997).
[15] L. K. Grover, in Proceedings of the 28th Annual ACM Symposium on the Theory of Computing (ACM, New York, 1996), pp. 212-219.

[16] D. Gottesman and I. L. Chuang, Nature (London) 402, 390 (1999).

[17] N. D. Mermin, Phys. Rev. Lett. 65, 1838 (1990).

[18] R. Laflamme, E. Knill, W. H. Zurek, P. Castati, and S. V. S. Mariappan, Philos. Trans. R. Soc. London, Ser. A 356, 1941 (1998).

[19] D. Bouwmeester, J.-W. Pan, M. Daniell, H. Weinfurter, and A. Zeilinger, Phys. Rev. Lett. 82, 1345 (1999); J.-W. Pan, D. Bouwmeester, M. Daniell, H. Weinfurter, and A. Zeilinger, Nature (London) 403, 515 (2000); M. Eibl, N. Kiesel, M. Bourennane, C. Kurtsiefer, and H. Weinfurter, Phys. Rev. Lett. 92, 077901 (2004).

[20] J.-W. Pan, M. Daniell, S. Gasparoni, G. Weihs, and A. Zeilinger, Phys. Rev. Lett. 86, 4435 (2001); N. Kiesel, C. Schmid, G. Toth, E. Solano, and H. Weinfurter, ibid. 98, 063604 (2007).

[21] Z. Zhao, Y.-A. Chem, A.-N. Zhang, T. Yang, H. J. Briegel, and J.-W. Pan, Nature (London) 430, 54 (2004).

[22] C.-Y. Lu, X.-Q. Zhou, O. Gühne, W.-B. Cao, J. Zhang, Z.-S. Yuan, A. Goebel, T. Yang, and J.-W. Pan, Nat. Phys. 3, 91 (2007).

[23] A. Rauschenbeutel, G. Nogues, S. Osnaghi, P. Bertet, M. Brune, J.-M. Raimond, and S. Haroche, Science 288, 2024 (2000).

[24] O. Mandel, M. Greiner, A. Widera, T. Rom, T. W. Hänsch, and I. Bloch, Nature (London) 425, 937 (2003).

[25] C. A. Sackett et al., Nature (London) 404, 256 (2000).

[26] See D. Leibfried, M. D. Barrrett, T. Schaetz, J. Britton, J. Chiaverini, W. M. Itano, J. D. Jost, C. Langer, and D. J.Wine- 
land, Science 304, 1476 (2004); and C. F. Roos, M. Riebe, H. Häffner, W. Hänsel, J. Benhelm, G. P. T. Lancaster, C. Becher, F. Schmidt-Kaler, and R. Blatt, ibid. 304, 1478 (2004), for the first multipartite entangled states using ion spins (for three electrons); see D. Leibfried et al., Nature (London) 438, 639 (2005) and H. Häffner et al., ibid. 438, 643 (2005), for sixpartite GHZ and W states.

[27] J. M. Elzerman, R. Hanson, L. H. W. van Beveren, S. Tarucha, L. M. K. Vandersypen, and L. P. Kouwenhoven, Lect. Notes Phys. 667, 25 (2005).

[28] G. Wendin and V. S. Shumeiko, e-print arXiv:cond-mat/ 0508729; Yu. Makhlin, G. Schön, and A. Shnirman, Rev. Mod. Phys. 73, 357 (2001).

[29] L. Quiroga and N. F. Johnson, Phys. Rev. Lett. 83, 2270 (1999); P. Zhang, C. K. Chan, Q. K. Xue, and X. G. Zhao, Phys. Rev. A 67, 012312 (2003).

[30] C. W. J. Beenakker, C. Emary, and M. Kindermann, Phys. Rev. B 69, 115320 (2004).

[31] S.-L. Zhu, Z. D. Wang, and P. Zanardi, Phys. Rev. Lett. 94, 100502 (2005).

[32] L. F. Wei, Y.-X. Liu, and F. Nori, Phys. Rev. Lett. 96, 246803 (2006); J. Q. You, X.-B. Wang, T. Tanamoto, and F. Nori, Phys. Rev. A 75, 052319 (2007).

[33] R. Migliore, K. Yuasa, H. Nakazato, and A. Messina, Phys. Rev. B 74, 104503 (2006).

[34] F. H. L. Koppens, C. Buizert, K. J. Tielrooij, I. T. Vink, K. C. Novack, T. Meunier, L. P. Kouwenhoven, and L. M. K. Vandersypen, Nature (London) 442, 766 (2006).

[35] J. R. Petta, A. C. Johnson, J. M.Taylor, E. A. Laird, A. Yacoby, M. D. Lukin, C. M. Marcus, M. P. Hanson, and A. C. Gossard, Science 309, 2180 (2005).

[36] A. O. Niskanen, K. Harrabi, F. Zoshihara, Y. Nakamura, S.
Lloyd, and J. S. Tasi, Science 316, 723 (2007); T. Hime, P. A. Reichardt, B L. T. Plourde, T. L. Robertson, C.-E. Wu, A. V. Ustinov, and J. Clarke, ibid. 314, 1427 (2006); J. Plantenberg, P. C. de Groot, C. J. P. M. Harmans, and J. E. Mooij, Nature (London) 447, 836 (2007).

[37] D. Loss and D. P. DiVincenzo, Phys. Rev. A 57, 120 (1998).

[38] M. Ciorga, A. S. Sachrajda, P. Hawrylak, C. Gould, P. Zawadzki, S. Jullian, Y. Feng, and Z. Wasilewski, Phys. Rev. B 61, R16315 (2000); J.M. Elzerman, R. Hanson, J. S. Greidanus, L. H. Willems van Beveren, S. De Franceschi, L. M. K. Vandersypen, S. Tarucha, and L. P. Kouwenhoven, ibid. 67, 161308(R) (2003).

[39] R. L. de Visser and M. Blaauboer, Phys. Rev. Lett. 96, 246801 (2006).

[40] M. Borhani and D. Loss, Phys. Rev. A 71, 034308 (2005).

[41] M. Blaauboer and R. L. de Visser, e-print arXiv:cond-mat/ 0609750.

[42] V. Coffman, J. Kundu, and W. K. Wootters, Phys. Rev. A 61, 052306 (2000).

[43] Michael A. Nielsen and Isaac L. Chuang, Quantum Computation and Quantum Information (Cambridge University Press, Cambridge, UK, 2000).

[44] For $N=2$ the fidelity is given by $F=\sqrt{1-A^{2}-B^{2}}$.

[45] We note in this context that proposals have been presented to replace the (slow) rotations by more of the (fast) interactions, but they require ancilla qubits and increase the number of required qubits by a factor of 3 ; see D. P. DiVincenzo et al., Nature (London) 408, 339 (2000).

[46] See, e.g., the chain of three quantum dots that has recently been realized by D. Schröer, A. D. Greentree, L. Gaudreau, K. Eberl, L. C. L. Hollenberg, J. P. Kotthaus, and S. Ludwig, Phys. Rev. B 76, 075306 (2007). 